\title{
Stimulated electromagnetic emission polarization under different polarizations of pump waves
}

\author{
E. D. Tereshchenko ${ }^{1}$, R. Y. Yurik ${ }^{1}$, and L. Baddeley ${ }^{2,3}$ \\ ${ }^{1}$ Polar Geophysical Institute, Murmansk, Russian Federation \\ ${ }^{2}$ University Centre on Svalbard, Longyearbyen, Norway \\ ${ }^{3}$ Birkeland Centre for Space Science, University of Bergen, Bergen, Norway \\ Correspondence to: R. Y. Yurik (roman.yurik@pgi.ru)
}

Received: 20 January 2014 - Revised: 18 January 2015 - Accepted: 9 February 2015 - Published: 5 March 2015

\begin{abstract}
The results of investigations into the stimulated electromagnetic emission (SEE) polarization under different modes of the pump wave polarization are presented. The present results were obtained in November 2012 during a heating campaign utilizing the SPEAR (Space Plasma Exploration by Active Radar) heating facility, transmitting in both O- and X-mode polarization, and a PGI (Polar Geophysical Institute) radio interferometer capable of recording the polarization of the received radiation. The polarization ellipse parameters of the SEE DM (downshifted maximum) components were determined under both O-mode and Xmode polarization of the pump waves. The polarization direction of the SEE DM component was preserved under different polarizations of the pump waves. Different polarizations of the pump waves have a different SEE generation efficiency. The intensity of the DM component is observed to be greater during O-mode pumping. In addition, the numbers of observed SEE features are also greater during O-mode pumping.
\end{abstract}

Keywords. Ionosphere (active experiments; plasma waves and instabilities) - radio science (ionospheric physics)

\section{Introduction}

Since the first registration of stimulated electromagnetic emission (SEE), during the heating experiments at Troms $\varnothing$ (Thidé et al., 1982), this phenomenon has been investigated using mainly amplitude methods. The SEE is a weak signal, limited across a frequency band of $\sim 150 \mathrm{kHz}$ around the pump frequency which is generated through the excitation of the ionospheric plasma by powerful radio emissions from a high-frequency (HF) heating facility. Observations of the SEE under different ionospheric conditions and different operation modes of the pump wave allow the characteristic features of the spectrum to be distinguished and the conditions affecting their generation to be identified.

It is known that the artificial generation of spectral components depends on several factors, such as the polarization of the pump wave, ionospheric conditions and the proximity of the pump wave to the local harmonic of the cyclotron frequency (gyroharmonic) of electrons (Stubbe et al., 1984; Leyser, 2001). The spectral width of the various components of the SEE may be a few hertz in the case of narrow-band components near the pump wave frequency and greater than $100 \mathrm{kHz}$ in the case of broadband radiation components.

An investigation into the SEE using radio-interferometric methods was started in 2000. This study has indicated that the processes involved in SEE generation are more complex than previously thought. In some cases the region of SEE generation may not coincide with the direction of maximum radiation of the pump wave (Carozzi et al., 2001; Tereshchenko et al., 2006). Additionally, Tereshchenko et al. (2012) showed that the emission of a discrete spectral feature of the SEE is not from a single-point location.

The study of the polarization characteristics of SEE near the fourth harmonic of the electron cyclotron frequency was carried out using the heating facility "Sura" in 1998. Studies have shown (Carozzi et al., 2001) that this type of measurement can be a new and informative diagnostic tool for SEE. In particular, it was found that part of the SEE spectrum has a low degree of circular polarization, which may be caused by the presence in the observed signal of overlapping different components with left and right polarizations and by the fact 
that the SEE signal comes from the spatial distribution of the generation region. Unfortunately, due to the sporadic nature of the study of data, many questions about the polarization of the SEE were not resolved in the course of the experiment.

The X-mode pumping has been performed in a number of experiments, with and without SEE diagnostics. Even in cases when no SEE diagnostics were used, the results bear on the physics of the ongoing processes in the present experiments (e.g. Thidé et al., 1983; Robinson et al., 1997). In this paper we present the results from a heating campaign utilizing the SPEAR (Space Plasma Exploration by Active Radar, Robinson et al., 2006) heating facility (located near Longyearbyen, Svalbard), which transmits circular polarization, and a radio interferometer capable of recording the polarization of the received radiation (located in Barentsburg, Svalbard).

Combined interferometric and polarization measurements allow us to investigate not only the general characteristics of the polarization of the received radiation but also to determine the radiation characteristics from different arrival directions.

\section{Description of the experiment}

The SPEAR heating facility is situated on Spitsbergen in the Svalbard archipelago $\left(78.15^{\circ} \mathrm{N}, 16.06^{\circ} \mathrm{E}\right)$. SPEAR is designed to carry out a range of space plasma investigations into the polar ionosphere and magnetosphere. The SPEAR site is located adjacent to the EISCAT (European Incoherent Scatter Scientific Association) Svalbard Radar (ESR). The SPEAR antenna system comprises a $6 \times 4$ array of fullwave, crossed dipoles, $16 \mathrm{~m}$ above the ground, with an antenna spacing of $48.4 \mathrm{~m}$, allowing the transmission of both linear and circularly polarized signals. The individual dipoles are rhombically broadened to allow operation between 4 and $6 \mathrm{MHz}$. The resulting beam has a quasi-elliptical cross section, with an average half-power width of $21^{\circ}$ along its major axis and $14^{\circ}$ along its minor axis. This results in an overall antenna gain of $21 \mathrm{~dB}$. Individual phase control provides beam steering within $\pm 30^{\circ}$ from zenith, at any azimuth, without significantly altering the antenna gain or introducing significant side-lobe signals. The beam may thus be pointed in directions which include vertical and field-aligned directions. SPEAR has a distributed transmitting and receiving system consisting of individual $4 \mathrm{~kW}$ solid-state transmitters connected to the antenna array. Each transmitter consists of a single driver stage and four individual $1 \mathrm{~kW}$ modules, the outputs of which are combined and fed to the antenna. In the current configuration 48 such transmitters are required. These are made up of 192 power-amplifier modules, 48 driver stages and a total of 240 power-supply units. Each transmitter contains an embedded controller and an individual direct digital synthesizer (DDS) which provides a transmit signal and the IF for the receiver front end.
For the polarization measurements of the SEE presented here, two channels of an HF inteferometer designed at the Polar Geophysical Institute (PGI) were employed. This interferometer has previously been used for the determination of the angle-of-arrival of SEE signals at the Sura (Nizhniy Novgorod, Russia), EISCAT (Troms $\varnothing$, Norway) and SPEAR heating facilities (Isham et al., 2005; Tereshchenko et al., 2006, 2008, 2011). The HF interferometer consists of an HF receiver with a high dynamic range and a band width of $300 \mathrm{kHz}$, a digital system for the conversion and recording of the received signal, and HF and IF (intermediate frequency) notch filters. The two channels of the HF receiver were used for the polarization measurements. The receiving antenna consists of a dipole antenna with $9 \mathrm{~m}$ masts which are oriented in the north-south and west-east geomagnetic planes. The data logging system uses two 16 bit analoguedigital converters (ADC) with two channels on each and a sampling frequency of $60 \mathrm{MHz}$. All channels of the ADC can work in synchronous and nonsynchronous modes. The HF and IF notch filters can be used for enlarging the dynamic range of the ADC. The receiving point was located at a PGI observatory (coordinates: $78.094^{\circ} \mathrm{N}, 14.208^{\circ} \mathrm{E}$ ) located about $40 \mathrm{~km}$ south-west of the SPEAR site.

\section{The calculation and data processing methods}

The coordinate system used for the Northern Hemisphere is shown in Fig. 1. On the figure the $x$ axis is directed toward the north, and the $y$ axis is directed toward the east. When using a dual-channel receiver for an orthogonal arrangement of dipole antennas oriented in the geomagnetic meridional (north-south) and zonal (east-west) planes (Fig. 1), the received signal of the quasi-monochromatic wave can be represented as

$$
\left\{\begin{array}{l}
E_{x}=E_{x 0} \cos \omega t \\
E_{y}=E_{y 0} \cos (\omega t+\Delta \varphi)
\end{array},\right.
$$

where $E_{x 0}$ and $E_{y 0}$ are the amplitude components of the electric oscillation and $\Delta \varphi$ is the phase difference between the oscillations in the meridional and zonal directions.

In those cases where $\Delta \varphi=n \pi(n=0,1,2 \ldots)$, the observed oscillations are linearly polarized. In the case of circular polarization, the phase difference $\Delta \varphi=(2 m-$ 1) $\pi / 2(m=1,2,3 \ldots)$.

The electric field vector in the horizontal plane describes an ellipse which can be represented by the equation

$\frac{x^{\prime 2}}{a^{2}}+\frac{y^{\prime 2}}{b^{2}}=1$,

where $x^{\prime}$ and $y^{\prime}$ represent coordinates in an orthogonal coordinate system oriented along the ellipse axes; $a$ and $b$ are the minor and major semi-axes of the ellipse:

$a^{2}=\frac{D^{2}}{2 A+2 \sqrt{A^{2}-D^{2}}}$, 


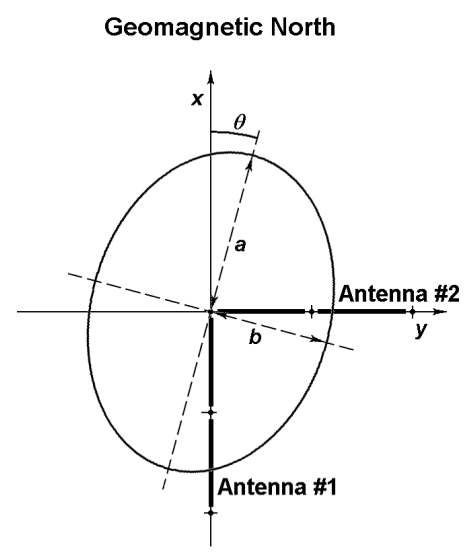

Figure 1. Orientations of the receiving antennas and the coordinate system.

$b^{2}=\frac{D^{2}}{2 A-2 \sqrt{A^{2}-D^{2}}}$.

In Eqs. (3)-(4), for brevity, the following notations were used:

$$
\left\{\begin{array}{l}
A=E_{x}^{2}+E_{y}^{2} ; \\
B=E_{y}^{2}-E_{x}^{2} ; \\
C=2 E_{x} E_{y} \cos \Delta \varphi ; \\
D=2 E_{x} E_{y} \sin \Delta \varphi
\end{array} .\right.
$$

In the horizontal plane the coordinate system $\left(x^{\prime}, y^{\prime}\right)$ is rotated relative to the original $(x, y)$ by the angle $\theta$ (see Fig. 1), which can be determined from the condition

$\operatorname{tg} 2 \theta=\frac{C}{B}$.

The set of parameters $A, B, C$ and $D$ in Eq. (5) is close to the set of Stokes parameters which was used by Carrozi et al. (2001).

To evaluate the shape of the polarization ellipse, the eccentricity parameter $(e)$ was used. This parameter describes the deviation of the shape of the ellipse from that of a circle. As $e$ approaches 0 , the polarization becomes more circular.

In our case, the eccentricity of the polarization ellipse is given by

$e=\sqrt{1-\frac{A-\sqrt{A^{2}-D^{2}}}{A+\sqrt{A^{2}-D^{2}}}}$

To determine $E_{x}, E_{y}$ and $\Delta \varphi$, the method of the spectral estimation of multichannel random processes has been used (Marple, 1987; Tereshchenko et al., 2006).

To analyse the spectrum of the observed signal, an average power spectral density (PSD), measured using two channels, was used, i.e.

$\operatorname{PSD}=A / 2=\frac{E_{x}^{2}+E_{y}^{2}}{2}$.
The phase difference between the electric field components in the zonal and meridional planes $(\Delta \varphi)$ was determined from the ratio between the real and imaginary parts of the complex parameter $E_{x y}$, i.e.

$\Delta \varphi=\operatorname{arctg}(D / C)=\operatorname{arctg}\left(\frac{\operatorname{Im}\left\{E_{x y}\right\}}{\operatorname{Re}\left\{E_{x y}\right\}}\right)$,

where $E_{x y}$ represents the scalar products of the two channels, $E_{x}$ and $E_{y}$.

The calculated absolute value of squared coherence (SC) of a two-channel system was used to differentiate between the radiated polarized components:

$\mathrm{SC}=\left|\frac{E_{x y}}{\sqrt{E_{x}} \sqrt{E_{y}}}\right|^{2}$.

In a real signal, where there is a noncorrelated noise, $\mathrm{SC} \neq 1$.

\section{The results}

This paper focuses on the observation of the SEE polarization parameters under different polarizations of pump waves. While the ADC utilized a sampling frequency of $60 \mathrm{MHz}$, each spectrum was obtained using an integration time of $2.2 \mathrm{~s}$ during one record. Twenty-seven series of observations (each series contains 70 records for $11 \mathrm{~min}$ of observation) which were obtained during the heating campaign from 13 to 17 November, were used for investigations. We selected four series which correspond time periods of similar ionospheric conditions and SPEAR operation mode. However, we have selected only that registration for which we observed a good SEE signal. The data were selected from four experiments carried out during 2012: two using O-mode polarization of the pump wave (13 November, 10:39:56 UTC, and 16 November, 09:33:26 UTC) and two using X-mode polarization (13 November, 11:25:26 UTC, and 16 November, 10:54:11 UTC).

The SPEAR radiated the pump waves along the geomagnetic field line (into magnetic zenith with an azimuth of $184^{\circ}$ and with an antenna main lobe inclination of $8^{\circ}$ south). The effective radiated power (ERP) was about $17 \mathrm{MW}$. The pumping series includes a 5 min pause before radiation was switched on and 10 min of radiation.

The possible mixture of both $\mathrm{O}$ and $\mathrm{X}$ polarizations in the transmission is a very important factor which should be taken into account for experiments and further interpretation of the polarization measurements. The most critical factors here are the phasing of each pair of crossed dipoles and the matching of the two antennas in power. This imbalance will give some leakage in the opposite mode, but it is a very small value and can be a few tens of decibels less than the main radiation. However, it was found early on that threshold for the SEE generation is about 5 MW ERP (Leyser et al., 1994; Frolov et al., 2000). Taking into account the low power of the SPEAR 

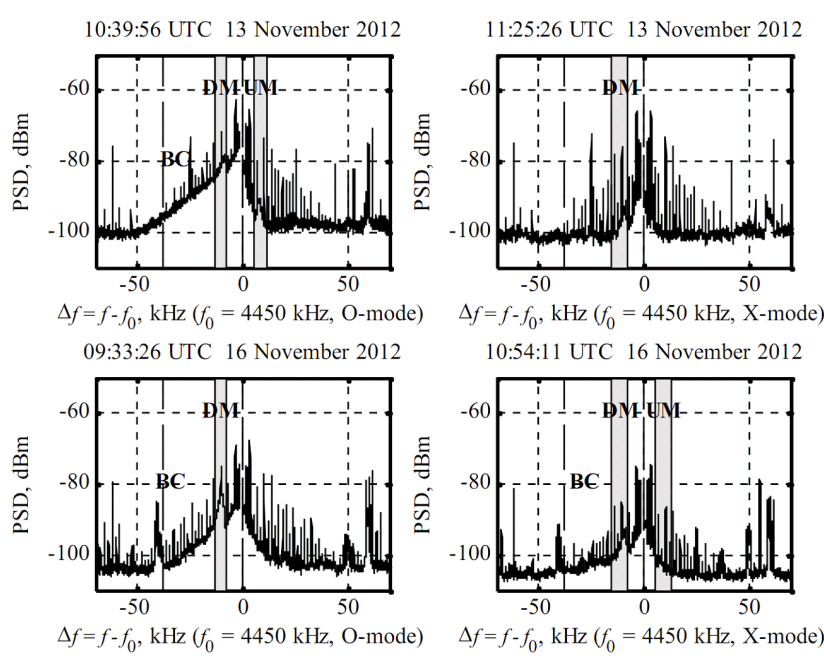

Figure 2. The power spectral densities (PSD) observed during the experiments.

radiation, we have assumed the absence of another polarization which was capable of exceeding the threshold of SEE generation.

It should be noted that SEE has been detected with less than $100 \mathrm{~kW}$ ERP of pump wave during preconditioning from previous pumping (e.g. Leyser et al., 1990). However, for the SPEAR location the horizontal plasma drift can remove the pump-induced irregularities from the pumpionosphere interaction region (Tereshchenko et al., 2011).

Figure 2 shows the PSD observed during the experiments conducted on the 13 (upper panel) and 16 (lower panel) November 2012 using the O-(left panel) and X-mode polarization (right panel) of the pump wave. The pump frequency is strongly attenuated by the notch filters and is not shown in the figures.

In all four cases, the DM (downshifted maximum) feature of the SEE spectrum was observed. The intensity of the DM was $20 \mathrm{~dB}$ above the noise floor in the case of O-mode polarization and about $10 \mathrm{~dB}$ in the case of the $\mathrm{X}$-mode polarization of the pump wave.

When using the pump wave with O-mode polarization, the $\mathrm{BC}$ (broad continuum) component is observed in the generated SEE spectrum. Spectral widths of the BC were up to $50 \mathrm{kHz}$ on 13 November and $40 \mathrm{kHz}$ on 16 November. During O-mode polarization the DM intensity is comparable between the 13 and 16 November; however, the BC intensity on the 16 November is decreased by $\sim 5 \mathrm{~dB}$.

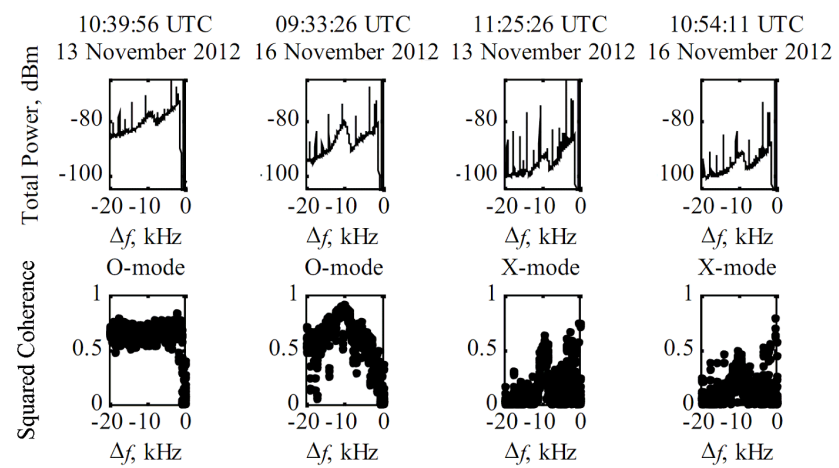

Figure 3. Plots of the total power (top row) and the squared coherence (bottom row).

Additionally, the SEE spectra observed during O-mode polarization of the pump wave on the 13 November showed an upshifted maximum (UM) feature at a frequency of $\Delta f=$ $8.6 \mathrm{kHz}$ above the pump wave frequency $(4.45 \mathrm{MHz})$ with an intensity of about $5 \mathrm{~dB}$ above the noise level.

When the X-mode polarization of the pump wave was used, the BC was not observed. The UM feature at a frequency of $9.3 \mathrm{kHz}$ with an intensity exceeding the floor noise level by $3 \mathrm{~dB}$ was observed on 16 November. In both cases, when a pump wave with $X$-mode polarization was used, the observed intensity of SEE was much smaller in magnitude when compared to the cases when a pump wave with O-mode polarization was used.

It should be noted that the polarization of the propagating waves is preserved only when the plasma density gradient is parallel or antiparallel to the geomagnetic field, which generally is not the case in the ionosphere. Thus, in general, all transmitted circularly polarized waves will be decomposed into two oppositely polarized waves (of different amplitudes) when entering into the ionosphere. In cases when wave propagation is not along geomagnetic field lines in the SPEAR beam, the transmitted circularly polarized wave will decompose into elliptically polarized O-mode and X-mode waves when entering into the ionosphere. For cases when SPEAR transmits pump waves along the geomagnetic field lines (slope angle equals $8^{\circ}$ and azimuth equals $184^{\circ}$ ), the angle between transmitted ray and geomagnetic field line can achieve $9^{\circ}$. Thus, at the edges, the pump wave pattern has some ellipticity.

For further analysis the spectral bandwidth from the pump wave frequency to $40 \mathrm{kHz}$ below the pump wave frequency was used (marked by the vertical dashed line in the plots in Fig. 2). Figure 3 shows plots of PSD (top row) and the coherence of the polarized component (SC) (bottom row) in the frequency range from -20 to $0 \mathrm{kHz}$ relative to the pump frequency. The PSD includes the DM feature. The analysis of the polarization parameters was carried out for the DM component because this spectral component of the SEE had been observed in all cases. 
Plots of the squared coherence (Fig. 3, bottom panels) show that, in all cases, the DM component of the SEE has a high degree of coherence and can be distinctly distinguished in the received signal except on 13 November 2012, when BC had a similarly high coherence. The absence of the highest values of coherence at 10:39:56 UT on 13 November 2012 (bottom row, first plot) could be caused by the more extended excitation region, which in turn gives less coherence because of the superposition of emissions at the receiving site. In the case of the pump wave with O-mode polarization, the observed $\mathrm{BC}$ feature of the SEE also has a high coherence.

The DM component of the SEE is located between -5 and $-15 \mathrm{kHz}$ from the pump wave frequency. In the case of O-mode polarization, a squared coherence level of 0.65 was observed. In the case of the X-mode polarization, a squared coherence level of 0.45 and 0.35 was observed for the experiments conducted on the 13 and 16 November respectively.

Figure 4 shows the phase difference $\Delta \varphi$, the angle of rotation of the polarization ellipse $\theta$ (upper panel) and eccentricity $e$ (lower panel) of the DM component of the SEE for all the selected four cases.

In all cases, the calculated eccentricity of the ellipse of polarization lies in the range of 0.8 to 0.9 , indicating an elliptical polarization of the observed signals, which arrive from the disturbed region according to the interferometer data. The angle between the major axis of the polarization ellipse and the meridional plane lies in the range from -10 to $20^{\circ}$, i.e. the polarization ellipse is focused mainly in the geomagnetic meridian plane. The slight differences in the values of the angle between the major axis of the polarization ellipse and the meridional plane indicate possible differences in the ionosphere or magnetic conditions at the time of observation, which lead to changes in the location of the pump-plasma interaction region and the conditions of the wave propagation from a one series of observations to another and from day to day.

The mean values of $\Delta \varphi$ are close to $90^{\circ}$ and were 100 and $87^{\circ}$ during O-mode polarization (10:39:56 UTC on 13 November and 09:33:26 UTC on 16 November respectively) and 79 and $118^{\circ}$ during $\mathrm{X}$-mode polarization (11:25:26 UTC on 13 November and 10:54:11 UTC on 16 November respectively).

The variation of $\Delta \varphi$ observed during periods of X-mode polarization is greater than that observed during O-mode polarization. It could be related to the weaker signal of the SEE under the X-mode pumping. Also, the variation of parameters may be due to differences in the ionospheric conditions during the observations as a result of high variability of ionospheric parameters in the polar cap.

\section{Discussion}

The utilization of the different pump wave polarizations leads only to a change in the efficiency of the generation of the SEE

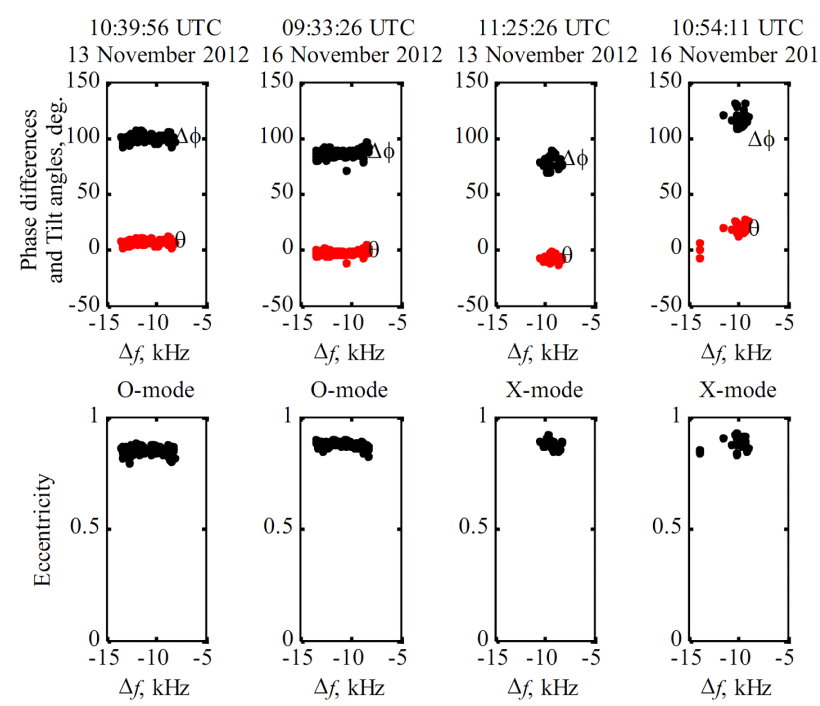

Figure 4. Plots of the phase difference $\Delta \varphi$, the angle of rotation of the polarization ellipse $\theta$ (upper panel) and eccentricity $e$ (lower panel) of the DM component of the SEE.

that was found during the early phase of SEE investigations (Thidé et al., 1983). The predominant O polarization of DM was preserved in both cases of the pump wave polarizations used. The preservation of the polarization direction of DM under different polarizations of the pump waves may indicate that the DM signals are generated in the same way.

The observed polarization ellipses of the DM signals were oriented in such a way that their major axes were in the meridional plane while, according to the geometry of the experiment, the arrival direction of the SEE signals was east. It is possible that the ellipticity of the polarization of the SEE was associated with the geometry of the signal propagation but was also affected by the spatial elongation of the generation region along the magnetic field. Thus, as the results show, in the magnetized ionospheric plasma the observed radiation of DM is elliptically polarized.

Although the theoretical concept of polarization was formulated mainly for quasi-monochromatic plane waves, the results show that this consideration is applicable to the study of the polarization characteristics of the particular spectral components (at least narrow maxima and peaks).

It is suggested that further studies of the polarization characteristics of the SEE should be carried out in combination with interferometric and polarization measurements in addition to other instrumentation utilized in the study of plasma dynamics. This would allow the determination of the radiation characteristics from different spatially localized sources.

\section{Conclusions}

Measurements of the polarization characteristics of SEE under different pump wave polarizations were undertaken and 
combined with simultaneous interferometric observations. In future experiments this method will allow the angles of SEE signal arrival from the emission region relating to specific polarization characteristics to be identified.

It was found that the SEE DM components had the same polarization direction (sign of the phase differences between electric oscillations in the meridional and zonal directions) for both O-mode and X-mode polarization of the pump wave. Different polarizations of the pump wave have a different efficiency regarding SEE generation. It was shown that the intensity of the DM component is greater under O-mode pumping. This result is consistent with previous results by Thidé et al., 1983. In addition, under X-mode pumping, the intensity and number of observed SEE features is less then under $\mathrm{O}$-mode pumping. The angle of rotation of the polarization ellipse, $\theta$, lies in the range of -10 to $20^{\circ}$ from the north.

Acknowledgements. L. J. Baddeley's research is supported by the Research Council of Norway/CoE under contract 223252/F5. SPEAR is supported by the Norwegian Research Council (grant 191628). The reported study was partially supported by RFBR, research project No. 15-05-02437 a.

Topical Editor K. Hosokawa thanks T. Leyser and one anonymous referee for their help in evaluating this paper.

\section{References}

Carozzi, T. D., Thide, B., Leyser, T. B., Komrakov, G., Frolov, V., Grach, S., and Sergeev, E.: Full polarimetry measuremnts of stimulated electromagnetic emissions: First results, J. Geophys. Res., 106, 21395-21407, 2001.

Frolov, V. L., Ermakova, E. N., Kagan, L. M., Komrakov, G. P., Sergeev, E. N., and Stubbe, P.: Features of the broad upshifted structure in stimulated electromagnetic emission spectra, J. Geophys. Res., 105, 20919-20933, 2000.

Isham, B., Hagfors, T., Khudukon, B., Yurik, R. Yu., Tereshchenko, E. D., Rietveld, M. T., Belyey, V., Grill, M., La Hoz, C., Brekke, A., and Heinselman, C.: An interferometer experiment to explore the aspect angle dependence of stimulated electromagnetic emission spectra, Ann. Geophys., 23, 55-74, doi:10.5194/angeo-2355-2005, 2005.

Leyser, T. B.: Stimulated electromagnetic emissions by highfrequency electromagnetic pumping of the ionospheric plasma, Space Sci. Rev., 98, 223-328, 2001.

Leyser, T. B., Thidé, B., Derblom, H., Hedberg, Å., Lundbrog, B., Stube, P., and Kopka, H.: Dependence of stimulated electromagnetic emission on the ionosphere and pump wave, J. Geophys. Res., 95, 17233-17244, 1990.
Leyser, T. B., Thide, B., Waldenvik, M., Veszelei, E., Frolov, V. L., Grach, S. M., and Komrakov, G. P.: Downshifted maximum features in stimulated electromagnetic emission spectra, J. Geophys. Res., 99, 19555-19568, 1994.

Marple, S. L.: Digital spectral analysis with applications, PrenticeHall, Inc., Englewood Cliffs, New Jersey, USA, 1987.

Robinson, T. R., Stocker, A. J., Bond, G. E., Eglitis, P., Wright, D. M., and Jones, T. B.: O- and X-mode heating effects observed simultaneously with the CUTLASS and EISCAT radars and low power HF diagnostics at Troms $\varnothing$, Ann. Geophys., 15, 134-136, 1997 ,

http://www.ann-geophys.net/15/134/1997/.

Robinson, T. R., Yeoman, T. K., Dhillon, R. S., Lester, M., Thomas, E. C., Thornhill, J. D., Wright, D. M., van Eyken, A. P., and McCrea, I. W.: First observations of SPEAR-induced artificial backscatter from CUTLASS and the EISCAT Svalbard radars, Ann. Geophys., 24, 291-309, doi:10.5194/angeo-24-291-2006, 2006.

Stubbe, P., Kopka, H., Thidé, B., and Derblom, H.: Stimulated electromagnetic emission a new technique to study the parametric decay instability in the ionosphere, J. Geophys. Res., 89, 75237536, 1984.

Tereshchenko, E. D., Yurik, R. Yu., Khudukon, B. Z., Rietveld, M. T., Isham, B., Belyey, V., Brekke, A., Hagfors, T., and Grill, M.: Directional features of the downshifted peak observed in HF-induced stimulated electromagnetic emission spectra obtained using an interferometer, Ann. Geophys., 24, 1819-1827, doi:10.5194/angeo-24-1819-2006, 2006.

Tereshchenko, E. D., Yurik, R. Yu., Yeoman, T. K., and Robinson, T. R.: First observations of stimulated electromagnetic emission in the ionosphere modified by the spear heating facility on Spitsbergen, Radiophys. Quantum El., 51, 858-861, 2008.

Tereshchenko, E. D., Yurik, R. Yu., Shadrin, S. A., Isham, B., Baddeley, L., and Rietveld, M. T.: The transformation of the stimulated electromagnetic emission spectra features under different heating pulse durations, in: Proceedings of the 15th EISCAT International Workshop/15th EISCAT International Workshop, Qingdao, 5-9 September 2011, 56, 2011.

Tereshchenko, E. D., Yurik, R. Yu., Rietveld, M. T., Isham, B., and Belyey, V.: The spatial features of the up- and downshifted maxima in stimulated electromagnetic emissions, Adv. Space Res., 50, 619-622, 2012.

Thidé, B., Kopka, H., and Stubbe, P.: Observations of Stimulated Scattering of a Strong High Frequency Radio Wave in the Ionosphere, Phys. Rev. Lett., 49, 1561-1564, 1982.

Thidé, B., Derblom, H., and Hedberg, Å.: Observations of stimulated electromagnetic emissions in ionospheric heating experiments, Radio Sci., 18, 851-859, 1983. 